\title{
Quelques points concernant la relation entre les normes juridiques et les normes éthiques dans la vie universitaire roumaine
}

\section{Cătălin Constantinescu-Mărunțel ${ }^{1}$}

Doctorant en Droit pénal

Chargé de TD, Théorie Générale du Droit/Doctrines Juridiques

Faculté de Droit, Université de Bucarest

Résumé : Dans le cadre de cet article nous essayerons de souligner premièrement les traits essentiels d'une norme juridique, en particulier les uns qu'on ne retrouve pas dans le cas des autres types de règles. Ensuite, nous chercherons les mécanismes utilisés par un system de droit et qui permettent la cohabitation et la collaboration entre les deux espèces ciblées: les normes juridiques et les normes éthiques. Afin d'avoir un point de vue complet, nous emploierons premièrement quelques exercices de comparaison, en utilisant plusieurs exemples similaires trouvés dans d'autres domaines. Nous allons vérifier sous quelles conditions on peut utiliser les sanctions juridiques dans des situations où une norme éthique a été violée.

Ceci dit, nous passerons à l'analyse des codes d'éthiques (ou de déontologie) utilisés par les universités roumaines. En raison de l'espace limité, nous utiliserons seulement les documents adoptés par l'Université de Bucarest, comme cette organisation fonctionne conformément à une architecture juridique-éthique représentative pour l'environnement universitaire roumain.

Au lieu des conclusions, nous ferons quelques propositions pour l'amélioration de ces codes, dans l'espoir que nous aiderons ainsi au développement de la culture éthique dans l'environnement académique roumain.

Mots-clés : normes, juridique, éthique, loi, sanctions, université.

Câteva puncte cu privire la relația dintre normele juridice și normele etice în mediul universitar român

Rezumat: În cadrul acestui articol vom încerca să subliniem în primul rând trăsăturile esențiale ale unei norme juridice, în particular pe cele pe care nu le întâlnim în cazul unor norme de altă natură. În continuare, vom cerceta mecanismele utilizate de un sistem de drept și care permit coabitarea și colaborarea între cele două specii vizate : normele juridice și normele etice. Cu scopul de a avea o perspectivă cât mai largă, vom folosi întâi câteva

\footnotetext{
${ }^{1}$ Cătălin Constantinescu-Mărunțel est doctorand en Droit pénal au cadre de l'Ecole doctorale de l'Université de Bucarest, Faculté de Droit. A partir de l'année universitaire 2014-2015 il est collaborateur de la même Faculté, en dirigeant les travaux préparatoires à la Théorie Générale de Droit et aux Doctrines Juridiques. Au sein de la société civile, il est membrecoordonnateur du Département pour la Justice Transitionnelle et Théorie Juridique au cadre du Centre pour l'Ethique, la Loi et l'Education (https://cede-cele.org/)
} 
comparații, pornind de la exemple comparabile din alte domenii. Vom verifica care sunt condițiile în care putem utiliza sancțiuni juridice în situațiile în care o normă etică a fost încălcată.

Acestea fiind discutate, vom trece la analiza codurilor etice (sau deontologice) utilizate de universitățile române. Din cauza spațiului limitat, vom utiliza doar documente adoptate de Universitatea din București, această organizație funcționând conform unei arhitecturi juridico-etice reprezentative pentru mediul universitar român.

În loc de concluzii, vom face câteva propuneri pentru ameliorarea acestor coduri, cu speranța că vom ajuta astfel la dezvoltarea culturii etice în mediul academic românesc.

Cuvinte cheie: norme, juridic, etic, lege, sancțiune, universitate.

\section{A few remarks about the relation between the legal norms and the ethical norms in the Romanian academic environment}

Abstract: In this paper we are beginning our research by underlining the essential characteristics of a legal norm, particularly those that one does not find in the case of other types of norms. Thereafter, we are examining the mechanisms employed by a legal system which allow the cohabitation and the collaboration between the two species that we are addressing: the legal norms and the ethical norms. In order to have a complete overview of the matter, we are utilising a few comparisons, relying on similar examples from other fields of study. We are researching under which conditions one may employ legal sanctions in cases where an ethical norm has been violated.

These being covered, we can continue by analysing the ethical (or deontological) codes used by the Romanian universities. For reasons of conciseness, we are limiting our scope to the documents adopted by the University of Bucharest. This can be done as this organisation functions on the basis of a legal and ethical normative architecture which is representative for the Romanian academic environment.

In place of conclusions, we are making a few proposals for the improvement of these codes. We are hoping that we are thus contributing to the evolving ethical culture in the Romanian universities.

Key words: norms, legal, ethical, act, sanctions, university.

\section{Introduction}

Une société dépourvue de règles ne peut pas exister. La nécessité de réglementation est un phénomène facile à repérer et bien connu, mais l'analyse ne peut pas être limitée à simplement observer qu'il existe un ensemble de normes. II faut explorer la nature (diverse) de ces normes et la manière dont elles interagissent au cadre de l'un ou de l'autre de domaines sociaux. On pourrait dire que ni les gens, ni les règles ne sont pas nées identiques. En réalité, ironiquement, les gens peuvent devenir égaux seulement par l'effet de normes. Donc, la loi, la moralité, la culture et l'économie donnent ensemble les lignes directrices pour les conduites humaines.

Dans le cadre de cet article nous essayerons de souligner premièrement les traits essentiels d'une norme juridique, en particulier les uns qu'on ne retrouve pas dans le cas des 
autres types de règles. Ensuite, nous chercherons les mécanismes utilisés par un system de droit et qui permettent la cohabitation et la collaboration entre les deux espèces ciblées : les normes juridiques et les normes éthiques. Afin d'avoir un point de vue complet, nous emploierons premièrement quelques exercices de comparaison, en utilisant plusieurs exemples similaires trouvés dans d'autres domaines. Nous allons vérifier sous quelles conditions on peut utiliser les sanctions juridiques dans des situations où une norme éthique a été violée.

Ceci dit, nous passerons à l'analyse des codes d'éthiques (ou de déontologie) utilisés par les universités roumaines. En raison de l'espace limité, nous utiliserons seulement les documents adoptés par I'Université de Bucarest, comme cette organisation fonctionne conformément à une architecture juridique-éthique représentative pour l'environnement universitaire roumain ${ }^{2}$. En tout cas, notre but n'est pas d'analyser en détail un code déontologique particulier. Si le lecteur veut une analyse en détail des similitudes et des différences entre les règlements adoptés par les universités roumaines, cette étude peut lui encore servir comme introduction dans le sujet ${ }^{3}$.

Au lieu des conclusions, nous ferons quelques propositions pour l'amélioration de ces codes, dans l'espoir que nous aiderons ainsi au développement de la culture éthique dans l'environnement académique roumain. En même temps, nous espérons que notre travail influencera d'autres auteurs et encouragera l'apparition de nouvelle recherche dans le domaine. Malheureusement, à ce moment, nous n'avons trouvé aucune étude juridique sur le sujet apparu dernièrement.

\section{Quelques mots concernant les normes juridiques et les normes éthiques}

Les normes juridiques sont définies comme "des règles de conduite, avec un caractère général, typique et obligatoire, régissant une relation sociale, règles qui sont exécutées volontairement par les citoyens ou par les autorités de l'État, ou en cas de manquement, sous la contrainte étatique." 4 II faut observer dès le début que ce type de norme a (inter alia) deux caractéristiques essentielles. D'une part, elles déterminent les conditions dans lesquelles se déroulent une ou plusieurs relations sociales, ces dernières devenant ainsi des relations juridiques ${ }^{5}$ (en roumaine, raportul juridic). D'autre part, la violation d'une norme juridique, contrairement aux violations d'autres types de normes, peut entraîner l'intervention des autorités publiques pour la correction de la situation ainsi née.

En fonction de leur source, les normes juridiques peuvent être divisées en deux catégories : légales et contractuelles. Les premières sont celles qui sont adoptées par l'État, à travers de ses autorités qui ont le droit ou l'obligation d'adopter des règles générales obligatoires. Les exemples sont multiples et bien connus : le Parlement, le Gouvernement, les Ministères, les Conseils Départementales etc. Dépendant du domaine social réglementé, toutes ces normes sont groupées en domaines du droit, comme le droit constitutionnel, le

\footnotetext{
${ }^{2}$ Emanuel Socaciu, Constantin Vică, Emilian Mihailov, Toni Gibea, Valentin Mureșan, Mihaela Constantinescu, Etică și integritate academică, Editura Universității din București, Bucarest, 2018, p. 50-51.

${ }^{3}$ Cependant, nous n'avons pas trouver une telle analyse comparative dans la littérature juridique roumaine.

${ }^{4}$ Simona Cristea, Teoria Generală a Dreptului, édition 3, C.H. Beck, Bucarest, 2019, p. 18.

${ }^{5}$ Dans le droit roumain il existe une différence entre la relation sociale et la relation juridique. La dernière est toujours une relation sociale réglementée par une norme juridique.
} 
droit civil, le droit pénal etc. Les sciences juridiques peuvent être partagées selon ce critère. Dès lors, les sciences juridiques sont "les sciences qui examinent un faisceau de relations sociales, ayant un objet de réglementation propre, méthode propre de recherche (méthode de domaine) et formulant principes de domaine." 6

A ce stade, il convient d'expliquer la différence entre la norme juridique et la source (formelle) de droit (en roumaine, izvorul formal de drept). Nous avons déjà mentionné que la norme juridique est une règle, une ligne directrice dans la mesure du possible claire, qui prévoit une ou plusieurs conduites qu'on doit adopter/entre lesquelles on doit choisir dans une situation particulière. Néanmoins, toutes ces normes sont immatérielles, existant seulement dans un plan formé par des idées et manquant une connexion palpable avec le monde matériel où on peut trouver l'individu. ${ }^{7} \mathrm{Ce}$ pont entre les deux espaces est représenté par la source formelle de droit, définie dans la doctrine roumaine comme "la manière dont sont exprimé les règles de droit, formes obligatoires qui contiennent préceptes pour les conduites et qui s'imposent dans l'ordre social avec une forme juridique, en bénéficiant de la force coercitive de l'État." ${ }^{8}$ En conséquence, du point de vue formel, les sources de droit sont les lieux où on peut trouver les normes juridiques.

En général, le droit roumain utilise les sources de droit suivantes : la loi écrite (ou l'acte normatif), le contrat normatif (ou le traité), la coutume et à titre exceptionnel, la jurisprudence. La doctrine juridique n'est jamais considérée une source de droit, bien que personne ne nie la grande influence qu'elle peut exercer sur la pratique. Naturellement, pas tous les domaines de droits utilisent les mêmes sources de droit de la même façon. Une logique particulière demande des instruments spécifiques. Cependant, à ce stade, on peut laisser de côté les domaines juridiques plus spécialisés, comme le droit fiscal, le droit de l'environnement ou même le droit pénal, pour nous concentrer mieux sur les relations de tous les jours. Ces dernières forment le champ d'application du droit civil. Conformément aux dispositions d'article 1 du Code civil roumain de 2011, „la loi, les coutumes et les principes générales de droit sont les sources de droit."

La différence entre une norme et sa source formelle est vraiment utile pour la compréhension de la manière dont une société ",jongle" avec un grand nombre de règles. ${ }^{9}$ Prenons un simple exemple : ne pas tuer. II est difficile de nier que cette règle a été toujours pertinente pour toute société. Aujourd'hui, on trouve cette norme juridique dans l'art. 102 par. (1) de la Constitution Roumaine de $1991^{10}$, dans l'art. 58 par. (1) du Code Civil de $2011^{11}$, dans l'art. 188 du Code Pénal ${ }^{12}$ etc. C'est-à-dire qu'une norme peut être exprimée au moyen de plusieurs sources formelles de droit, avec ou sans des nuances nécessaires pour la contextualisation.

C'est dans cet esprit qu'on doit observer que les normes juridiques n'apparaissent pas du néant ou sur un coup de tête du destin. Elles sont le produit d'une série des facteurs culturels, économiques, politiques etc. ${ }^{13}$ Une réalité qui nous donne la chance de

\footnotetext{
${ }^{6}$ S. Cristea, Teoria Generală a Dreptului, p. 6.

${ }^{7}$ Dan Claudiu Dănișor, Ion Dogaru, Gheorghe Dănișor, Teoria generală a dreptului, C.H. Beck, Bucarest, 2006, p. 32-40.

8S. Cristea, Teoria Generală a Dreptului., p. 37.

${ }^{9}$ Humberto Avila, Theory of Legal Principles (Law and Philosophy Library), Springer, 2007, p. 6 - 7.

${ }^{10}$ "Le droit à la vie, ainsi que le droit à l'intégrité physique et mentale sont garantis."

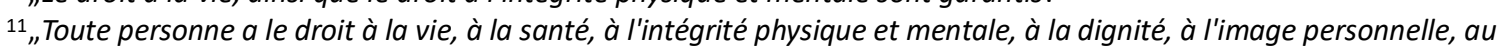
respect de la vie privée, ainsi que des autres droits reconnus par la loi."

12 "Le meurtre d'une personne est puni par prison de 10 à 20 années et l'interdiction de la jouissance de certains droits."

${ }^{13}$ Lidia Barac, Elemente de Teoria Dreptului, All Beck, Bucarest, 2001, p. 13-15.
} 
mentionner les sources matérielles de droit, à leurs tours déterminantes pour la compréhension de la logique du système de droit roumain. Elles représentent „les conditions préexistantes par rapport au droit, comme le cadre économique, social, politique, culturel, idéologique, qui sont imposées au législateur dans le processus de la création du droit." ${ }^{14}$ Alors, on pourra affirmer que le droit évolue afin de répondre à des exigences existant au niveau de la société.

Ces besoins sociaux sont très divers et ils correspondent aux particularités du chaque secteur d'activité. II est possible que l'État ait besoin de collecter plusieurs taxes ou il est possible qu'il soit nécessaire dans une région de faciliter les interactions entre l'administration publique et les citoyens dans une certaine langue. D'habitude on parle des situations concrètes, mais ça ne signifie pas qu'on ne peut pas rencontrer des autres scénarios. Par exemple, un changement législatif pourrait être généré par l'augmentation en importance d'une norme préexistant d'autre nature ${ }^{15}$. Un tel cas implique le besoin de changer les heures de travail pendant la période du Ramadan dans une communauté où il y a beaucoup de musulmans. En d'autres termes, il y a des situations où l'État pourrait être intéressé de donner un caractère juridique à une règle ou à un principe d'origine religieuse, comme la plupart des citoyens ont une certaine foi. Par conséquent, pour ces gens, la norme religieuse sera doublée par une norme juridique identique. La source formelle de droit de la dernière sera l'acte normatif adopté par les autorités publiques, tandis que la première garde sa source originale de nature religieuse (musulmane dans notre exemple hypothétique).

L'idée centrale exposée dans le dernier paragraphe est réplicable à la fois au niveau central et par rapport aux autres types de normes. Plus important peut-être, elle peut marquer le désir du législateur de limiter les domaines réglementés par lui-même en utilisant des normes juridiques. Par exemple, même si le droit civil est l'un des plus riches domaines du droit, il est aussi vrai que les relations sociales qui forment son champ d'application sont si diverses qu'elles peuvent devenir très difficile de règlementer après un certain point. C'est pourquoi la Roumanie a déjà une longue tradition juridique de reconnaître qu'il y a certains domaines où il est mieux d'utiliser directement les normes sociales préexistantes, comme les coutumes locales qui trouvent leur origine dans la moralité ${ }^{16}$.

L'exemple le plus connu qu'on peut donner afin de concrétiser cette idée est la notion de bonne foi. Article 14 paragraphe (1) du Code civile établit que "toute personne physique ou morale est obligée d'exercer ses droits et d'exécuter ses obligations civiles en toute bonne foi, en accord avec l'ordre publique et les bonnes mœurs." ${ }^{17}$ Même si la notion de bonne foi est cruciale pour la compréhension de la logique du droit civile, art. 14 est le seul texte où le législateur du 2011 a essayé de le définir. Malheureusement, comme

\footnotetext{
${ }^{14}$ S. Cristea, Teoria Generală a Dreptului., p. 37.

${ }^{15}$ Le législateur doit harmoniser les normes juridiques par rapport aux normes d'autres natures. On peut consulter loan Muraru, Elena Simina Tănăsescu, Drept constituțional și instituții politice, édition 13, C.H. Beck, Bucarest, 2008, p. 7.

${ }^{16}$ Cette idée est aussi valable pour la période du régime communiste, mais nous voulons préciser que cela ne signifie pas que nous sommes d'avis que le régime communiste a été un régime politique morale. Bien au contraire, à notre avis le régime communiste roumain a été un régime criminel. Pour une explication agrée par ce régime de la notion de justice, on peut consulter lonel Cloșcă, Ioan Pohonțu, Ion Stanca, Dicționar Juridic pentru Militari, Editura Militară, București, 1975, p. 121.

17Le Code Civil Roumain de 2011 se trouve (en roumain) à l'adresse http://legislatie.just.ro/Public/DetaliiDocument/ 109884, visitée le 10 Décembre 2020.
} 
beaucoup d'autres définitions employées par le droit roumain à ce jour, cette définition est aussi un peu circulaire. Pour comparaison, il nous semble plus juste et adéquate cette manière de la définir, trouvée dans la littérature étrangère : „la bonne foi signifie la prise par un individu d'une conduite pour une raison positive, en se fondant sur un sentiment de légalité ou afin d'exécuter une obligation, dans le cas où l'obligation représente la nécessité d'agir en vue de respecter la loi."18

Avant de passer à la section suivante, on pourrait tirer trois conclusions sur la façon dont les normes juridiques fonctionnent dans le système de droit roumain. Premièrement, ces règles sont les seules normes adoptées par les autorités publiques. En outre, elles sont les seules normes pour lesquelles on peut appeler directement à la force coercitive de l'État en cas de violation. Deuxièmement, les normes juridiques peuvent reproduire le contenu des normes d'une autre nature. Également, elles peuvent être complétées par des normes d'une autre nature. Tout cela étant dit, on peut formuler la troisième idée. Les normes d'autre nature peuvent fonctionner et produire des effets concrets indépendamment ou à travers des normes juridiques. ${ }^{19}$ Naturellement, cette notion est utile pour le sujet de cet article, comme elle s'applique aussi pour les normes éthiques.

Dans ce cadre, il devient clair que les dernières peuvent guider le comportement d'une personne dans l'espace publique si elles ne sont pas en conflit avec les normes juridiques ou si les normes juridiques prescrivent qu'on est dans un tel cas. Les deux hypothèses peuvent être mieux expliquées si on imagine un carré avec les faces bien bordées, mais avec une surface intérieure transparente. Les faces sont le cadre général formé par les normes juridiques, à l'intérieur duquel on déroule les activités quotidiennes. La première et la plus simple hypothèse est basée sur le fait que, dans une démocratie moderne, le législateur ne veut pas et, en tout cas, ne devrait jamais réglementer chaque relation sociale. Au contraire, une telle intention tend à caractériser les régimes totalitaires et, dans une certaine mesure, ceux autoritaires.

Il semble bien qu'à l'intérieur du carré existe un vide normatif, mais c'est entièrement une apparence. ${ }^{20}$ Dans cet endroit se manifestent les normes d'une autre nature, telles que celles éthiques, culturelles ou économiques. Par ailleurs, on doit préciser que la Roumanie est une société assez diverse, avec beaucoup de minorités culturelles, religieuses, historiques etc. Par conséquent, il y a un certain nombre d'ensembles de règles de même nature qui se manifestent simultanément. Par exemple, les normes religieuses d'origine Chrétienne Orthodoxe doivent coexister avec les normes religieuses d'origine Islamique, Mosaïque, Chrétienne Catholique etc. II en va de même des normes morales parce qu'elles peuvent différer entre une ville et une autre ou entre une famille et une autre. Dès lors, il peut devenir difficile de déterminer le numéro et le contenu des ensembles de normes sociales dans une communauté plus large. A notre avis, étant donné la composition ethnique et religieuse de la Roumanie, on pourra soutenir que l'idée reste valide dans un tel pays.

Dans ce cas, si les personnes sont guidées par de séries différentes de normes, le législateur peut les utiliser pour compléter le cadre légal, mais il a besoin d'établir de

\footnotetext{
${ }^{18}$ Simon Blackburn, Ethics. A very short introduction (Very Short Introductions), Oxford University Press, Oxford, 2001, p. 102.

${ }^{19}$ Simona Cristea, Doctrine Juridice, édition VII, C.H. Beck, Bucarest, 2017, p. 142.

${ }^{20}$ Nicolae Popa, Ion Dogaru, Gheorghe Dănișor, Dan Claudiu Dănișor, Filosofia dreptului. Marile curente, édition II, C.H. Beck, Bucarest, 2007, p. 344-345.
} 
manière exacte comment le système va fonctionner. Par ailleurs, il aurait violé l'un des principes fondamentaux de l'élaboration des lois, notamment le principe de l'accessibilité et de l'économie de moyens dans l'élaboration normative. Selon la littérature juridique, les normes doivent être formulées dans une manière claire, brève et accessible pour le destinataire ${ }^{21}$. A défaut, la règle peut devenir inapplicable, mais il est également possible de créer une alternative tout aussi problématique. Si une personne est accusée d'avoir violé une norme confuse, cela signifie que le juge reçoit un pouvoir discrétionnaire quant à l'interprétation de cette norme ${ }^{22}$.

Comment fonctionnent ces normes de référence peut être facilement illustré par rapport à une des mieux réglementées professions en Roumanie, la profession d'avocat. L'activité des avocats se déroule conformément aux dispositions de la Loi no. 51/1995 pour I'organisation et l'exercice de la profession d'avocat ${ }^{23}$, mais cet acte normatif ne fonctionne pas indépendamment. La loi est complétée par le Statut de la profession d'avocat ${ }^{24}$, par le Code déontologique de l'avocat roumain ${ }^{25}$ et par le Code déontologique des avocats de I'Union Européenne ${ }^{26}$. Donc, nous remarquons qu'il y a quatre séries de normes applicables, deux de nature juridique et deux de nature éthique, mais l'architecture repose sur les règles inclues dans la Loi no. 51/1995.

Le rôle de la déontologie professionnelle dans la vie d'un avocat est indiqué par les articles 55 par. (2) d) et 63 par. (1) f) Loi no. 51/1995. Les organes de direction de la profession d'avocat (soit au niveau de barreau, soit au niveau national) sont compétents pour proposer, respectivement pour établir le contenu du code d'éthique. En d'autres termes, le législateur a mis en place le cadre légal par l'adoption d'un acte normatif, mail il a laissé aux destinataires de la loi la liberté d'enrichir ce cadre avec des normes avec une force normative inférieure, soit de nature juridique, soit d'autre nature.

Quand même, il était dans l'intérêt de l'État roumain de permettre à ces normes complémentaires de nature éthique de produire des effets juridiques concrets. Cela signifie qu'il était nécessaire d'imposer aux avocats une obligation légale d'obéissance par rapport au Code de déontologie. Conformément à l'article 2 par. (1) de la Loi no. 51/1995, „dans l'exercice de la profession, l'avocat est indépendant et il est assujettie seulement à la loi, au statut de la profession et au code déontologique". Puis, la règle est renforcée par l'article 13 par. (6), où on prévoit que les avocats étrangers sont aussi assujettis au même code d'éthique. En plus, art. 38 par. (3) établit que les règles de déontologie s'appliquent également aux relations nées entre l'avocat et sa clientèle.

Les mêmes idées sont reprises et développées dans le Statut de la profession d'avocat. Celui-ci vise à créer une deuxième série de norme juridiques avec une force normative inférieure par rapport à la Loi no. 51/1995. Néanmoins, elles ont l'avantage de pouvoir être adoptées, modifiées ou abrogées plus facilement, comme tous ces procédures restent du ressort des organes de direction de la profession. En plus de reprendre les prescriptions susmentionnées, le Statut introduit quelques-unes nouvelles. Par exemple, art.

${ }^{21}$ S. Cristea, Teoria Generală a Dreptului, p. 72-44.

${ }^{22}$ Simona Cristea, Doctrine Juridice, p. 126-133.

${ }^{23}$ Publiée dans le Monitorul Oficial no. 440 de 24 Mai 2018.

${ }^{24}$ Adopté par la Décision du Conseil de I'Union Nationale des Barreaux de Roumanie no. 64/2011, publié dans le Monitorul Oficial no. 898 de 19 Décembre 2011.

${ }^{25}$ Adopté par la Décision du Conseil de l'Union Nationale des Barreaux de Roumanie no. 268/2017.

${ }^{26}$ Adopté dans la Session Plénière de la C.C.B.E. de 28 Octobre 1988, puis modifié au cadre des session plénières de 28 Novembre 1998, 6 Décembre 2002 et 19 Mai 2006. 
231 par. (2) nous indique que „par responsabilité professionnelle on entend la réparation de dommages effectives subis par le client et provenant de l'exercice de la profession au nonrespect des dispositions de la Loi, du présent Statut et des règles déontologiques." En ces conditions, la Loi no. 51/1995 et le Statut forment l'ensemble de normes principales qui régissent l'activité des avocats. Cela nous donne la possibilité de mieux comprendre quel est le rôle joué par les normes éthiques.

Conformément à l'article 5 du Code déontologique de l'avocat roumain, celui-ci „établit les principes essentiels et les règles de conduite que l'avocat roumain doit respecter, tant dans l'exercice de la profession d'avocat que dans sa vie publique et privée. Ces principes et règles constituent la base de toute interprétation des réglementations professionnelles, ainsi que de toute initiative de réglementation professionnelle soit de modification ou de complément aux dispositions existantes." 27 En outre, conformément à l'article 1.3.1. du Code déontologique des avocats de l'Union Européenne, le processus d'élaboration de ces normes au niveau de I'UE „a essentiellement a pour objectif l'atténuation des difficultés qui découlent de l'application d'une double déontologie."28

Nous ne reprendrons pas tous les prescriptions contenues par les deux codes, mais il faut faire une petite observation cruciale. Aucun des deux documents n'est fait pour établir des lignes directrices claires pour des situations concrètes qu'on peut vraiment rencontrer au cadre de la profession d'avocat. Toutes ces normes ont plutôt les traits normaux pour les principes. Pour cette raison, on peut seulement difficilement affirmer que leur destinataire peut les utiliser exclusivement afin d'organiser sa vie professionnelle. Par conséquent, nous sommes d'avis que, dans un tel système, les normes éthiques ont d'abord le rôle de faciliter la compréhension des normes juridiques. Etant donnée leur haut niveau de généralité, il est difficile pour eux d'être suffisantes pour imposer des règles de conduite supplémentaires. Néanmoins, à l'arrière-plan, il nous reste les cas pour lesquels on n'a aucune norme juridique. Dans un tel scénario, un code de déontologie peut aider dans le processus de trouver une solution, directement ou par analogi $e^{29}$. Cela sauve partialement le rôle pratique des codes de déontologie utilisés par les avocats mais, malgré tous ces arguments, le fait qu'elles sont très générales continue de nous empêcher d'identifier dans les deux codes une norme qu'on peut utiliser indépendamment comme base pour l'application d'une sanction disciplinaire.

A la lumière de ce qui précède, nous pouvons déjà remarquer que les normes éthiques fonctionnent seulement si le système normatif respecte au minimum deux principes. Premièrement, elles doivent être applicables aux lignes de conduite concrètes. C'est-à-dire qu'il convient de les formuler d'une manière moins générale, en mesure d'offrir une prescription claire pour une situation qu'on pourra rencontrer dans la vie quotidienne, professionnelle ou pas. Deuxièmement, un code déontologique ne doit pas être juste une doublure de l'acte normatif, qu'il soit de nature légale ou contractuelle. Si la règle éthique a le même contenu que la règle juridique, la règle éthique est inutile pour la relation juridique. Donc, il faut créer une architecture normative qui utilise en même temps toutes les deux séries de normes de façon complémentaire. Nous examinerons en détail cette idée dans les paragraphes suivants.

\footnotetext{
${ }^{27} \mathrm{https}: / /$ www.unbr.ro/publicam-hotararea-consiliului-unbr-nr-26817-iunie-2017-prin-care-se-aproba-codul-deontologic-alavocatului-roman-prevazut-in-anexa/, consulté le 17 Décembre 2020.

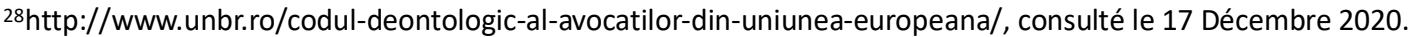

${ }^{29}$ |l est bien sûr nécessaire d'éviter l'utilisation de l'analogie aux dépens de la personne accusée.
} 
Comme nous n'avons pas l'espace nécessaire pour analyser toutes les dispositions du code, il faut choisir une série de normes représentative. Au cadre de la sous-section III.4 du Code déontologique de l'avocat roumain, intitulée le principe d'éviter les conflits d'intérêt, on trouve les normes mises en place pour prévenir telles situations. La notion de conflit d'intérêt n'est jamais définie, mais on pourra l'inférer sur la base des paragraphes (1) - (5). Ces dispositions mentionnent quelques circonstances où un tel conflit pourra apparaître. Par exemple, on voit que "I'avocat ne peut pas assister ou représenter un client dans la mesure où les intérêts du client sont soit pourraient être en conflit avec les intérêts des autres clients ou de l'avocat." On ne sait pas si le texte fait référence à tous les intérêts possibles de l'avocat ou seulement à certains intérêts, comme les uns de nature économique, politique soit professionnelle. Dans une manière similaire, par. (2) établit que "I'avocat ne peut pas ne peut pas plaider contre la partie qui l'a consulté au préalable au sujet des aspects litigieux concrets de la cause." Autant que dans l'exemple précédent, le pivot de l'ensemble du jugement n'est jamais défini. Quels sont les aspects litigieux concrets ? Est-ce qu'on pourra considérer comme ayant une telle nature tous les aspects qui ont une liaison avec le litige, juste la stratégie procédurale ou peut-être seulement les éléments de la stratégie qui ont été utilisés au cadre du litige ? Il est évident que l'élaboration des normes par le recours à des termes vagues peut avoir une seule finalité : I'application avec de très grandes et inutiles difficultés.

Prenons, pour comparaison, quelques codes éthiques de grandes entreprises et examinons la manière dont elles ont essayé d'éviter ces problèmes. Il faut remarquer du début que cette analyse nous permettra de présenter un autre système au cadre duquel les normes juridiques peuvent être complétées par les normes éthiques. Donc, il faut préciser qu'il est possible d'avoir des règles éthiques qui ont la capacité de produire des effets juridiques en vertu des règles juridiques contractuelles, à leurs tours adoptées en respectant les normes juridiques légales applicables.

La troisième section du Code de conduite de Google ${ }^{30}$, intitulée La prévention des conflits d'intérêt, pourrait être un très bon exemple. Cela commence avec deux paragraphes qui visent à expliquer la notion de conflit d'intérêt en créant un rapport clair entre la notion d'intérêt privé d'un salarié et les intérêts de l'entreprise. Puis, il nous est indiqué une procédure préalable mise en place pour la prévention de telles situations. En résumé, le collaborateur peut poser des questions à son chef ou au département Ethique et Conformité (Ethics and Compliance). Ensuite, la notion de conflit d'intérêt est expliquée, illustré avec des exemples et prévenue en fournissant des solutions pratiques pour 11 scénarios qui peuvent apparaître au cadre de l'activité de Google. Le dernier point de la section prévoit l'obligation du département susmentionné d'informer périodiquement la direction de l'entreprise concernant les types de conflits d'intérêt identifiés.

Starbucks a choisi une approche similaire, en traitant la notion dans une section distincte de son code de conduite ${ }^{31}$. On indique du début que "un conflit d'intérêt existe quand un intérêt ou une activité personnelle interfère soit créé l'impression d'une interférence $e^{\prime \prime 32}$ par rapport aux obligations du salarié envers l'entreprise. Puis on précise clairement qu'il a l'obligation de les analyser au niveau central et de les rapporter

\footnotetext{
30https://abc.xyz/investor/other/google-code-of-conduct/, consulté le 18 Octobre 2020.

${ }^{31}$ Starbucks, Business Ethics and Compliance. Standards of Business Conduct, p. 15 - 16. On peut trouver le code à https://globalassets.starbucks.com/assets/eecd184d6d2141d58966319744393d1f.pdf, consultée le 28 décembre 2020. 32/dem, p. 15.
} 
périodiquement aux autorités compétentes. Contrairement au Google, dans ce cas on préfère les exemples pratiques. En fait, on trouve de normes secondaires seulement dans la matière d'offrir ou de recevoir de cadeaux de la part des partenaires externes. Pour ce cas, le code établit une limite de la valeur annuelle acceptable pour tels cadeaux et il donne plusieurs solutions pour éviter de violer ces normes. De toute façon, il existe une obligation clairement précisée pour chaque employé de consulter un département spécialisé dans un tel scénario problématique (Business Ethics and Compliance) et de ne pas s'empresser de commettre quelque chose que pourrait créer un conflit d'intérêt.

Pour le troisième exemple nous avons choisi un code développé dans le secteur privé, mais qui traite le conflit d'intérêt d'une manière plus concise. Le but est de découvrir quelles sont les normes maintenues dans le code, même si l'auteur a préféré d'avoir une série brève de règles. Dès lors, le Code de conduite de Deloitte - Canada ${ }^{33}$ est un bon sujet d'analyse. II commence par préciser que „les conflits d'intérêts - qu'ils soient réels ou perçus - se produisent lorsqu'un jugement professionnel est affecté par des influences extérieures." ${ }^{34}$ Par la suite, le code indique quels sont les effets qui peuvent être produits par une telle situation et que chaque employé a l'obligation de prévenir un tel scénario. Puis, comme dans le cas du Code déontologique de l'avocat roumain, on trouve six hypothèses possibles concrètes et les conduites qu'on est obligé d'adopter dans chaque cas. Malheureusement, il y a beaucoup de notions qui sont utilisées, mais elles ne sont pas définies. Par exemple, on sait qu'un salarié doit s'abstenir d'avoir des activités sociales avec le client, mais ils ne nous sont jamais indiquées les limites de cette obligation. En plus, le code de Deloitte - Canada n'indique pas une structure spécialisée qu'on peut contacter si on veut savoir comment agir afin de prévenir un conflit d'intérêt. Néanmoins, le code établit l'obligation de chaque employé d'annoncer son supérieur si un tel conflit peut apparaître.

A la lumière de ce qui précède, nous pouvons déjà souligner quelques points concernant la dynamique qu'on peut observer entre les normes juridiques et les normes éthiques dans le système roumain de droit :

1) la conformité avec les normes éthiques peut être imposée au moyen des normes juridiques légales, comme une loi sur l'éducation, ou au moyen des normes juridiques internes, comme les règlements applicables au niveau d'une organisation. A notre avis, il est préférable d'utiliser tous les deux mécanismes ;

2) il est important de faire une mention à ce stade, parce qu'on doit savoir du début quel est le but précis du code de déontologie. Si notre but est d'utiliser les normes de nature éthiques comme base pour l'application des sanctions prévues par les normes juridiques, on doit formuler la règle éthique d'une manière conforme aux exigences juridiques. Si notre but est seulement de formuler un document qui exprime nos idéaux, mais sans valeur pratique, nous pouvons formuler les normes même d'une manière poétique.

Les normes éthiques peuvent avoir un objectif étroit ou un objectif étendu. Dans le premier cas, les normes éthiques aide seulement à l'interprétation de la norme juridique. Dans le deuxième cas, un code de déontologie peut régir la conduite de la personne dans un cadre spécifique.

\footnotetext{
${ }^{33}$ Deloitte, Code d'éthique, p. 10. Le Code peut être trouvé à

https://www2.deloitte.com/content/dam/Deloitte/ca/Documents/about-deloitte/ca-fr-apropos-code-dethique.pdf, consulté le 18 Octobre 2020.

${ }^{34} \mathrm{Ibidem}$.
} 
3) si on veut utiliser les normes éthiques pour régir les conduites humaines, on a besoin de bien définir les notions employées et de clairement préciser quelles sont les obligations imposées par cette voie. II est nécessaire d'éliminer les phrases vagues, équivoques, parce qu'on ne peut pas sanctionner la violation d'une règle exprimée tellement. En plus, une bonne pratique est la formulation d'une règle concise et puis de donner des exemples afin d'expliquer cette règle.

4) il est également utile de créer un mécanisme consultatif et de le mettre à la disposition des employés. Cela permet l'imposition de l'obligation de chaque employé de consulter la structure responsable de l'application du code déontologique quand il ou elle a des questions concernant une situation problématique. Une telle obligation doit être de nature juridique, comme il est mieux si elle est basée directement sur les dispositions du contrat de travail.

\section{Les codes déontologiques universitaires}

Conformément à l'article 124 de la Loi pour l'Education Nationale ${ }^{35}$, les universités, soit qu'elles sont publiques, soit qu'elles sont privées, porte la responsabilité publique pour leur activité. Inter alia, cela signifie que les universités doivent „respecter la politique d'équité et d'éthique universitaire, contenue dans le Code d'éthique et de déontologie professionnelle approuvé par le Sénat universitaire." Puis, l'article 130 de la même loi établit qu'un tel code doit régir au minimum les sujets suivants :

„- quand peuvent apparaître les conflits d'intérêts et les incompatibilités ;

- la disposition que les personnes ayant de liens comme les époux ou les proches jusqu'au troisième degré ne peuvent pas avoir au même temps des fonctions si cela signifie que un se trouve dans un poste d'encadrement, contrôle, autorité ou évaluation institutionnelle à n'importe quel niveau dans l'université et ne peuvent pas être nommés pour faire partie des commissions de doctorat, des commission d'évaluation ou des commissions de concours si les décisions prises par ces commissions touchent ces personnes ayant de liens comme les époux ou les proches jusqu'au troisième degré ;

- les mesures d'enseignement, administratives et techniques qui sont pris afin de garantir l'originalité des thèses de licence, de master et de doctorat, des articles scientifiques et des autres travaux similaires, ainsi que les sanctions correspondantes."

Néanmoins, le texte législatif n'offre plus d'information concernant ce sujet. A notre avis, cela veut dire que le législateur roumain n'a pas voulu décider comment on doit intégrer un tel code parmi les normes juridiques applicables dans ce domaine. Comme les universités sont autonomes en Roumanie, il est attendu de leur donner la liberté nécessaire de décider eux-mêmes. En tout cas, cela signifie aussi que nous pouvons passer à l'analyse de quelques documents adoptés au niveau universitaire.

La Charte de I'Université de Bucarest (Carta Universității din București) ${ }^{36}$ prévoit les plus importantes normes juridiques adoptées au niveau de cette organisation. De façon prévisible, ces normes sont complémentaires et ont une force normative inférieure par rapport à la Loi pour l'Education Nationale. If faut mentionner aussi qu'on trouve ici des

\footnotetext{
${ }^{35}$ La Loi pour l'Education Nationale no. 1 de 5 Janvier 2011, publiée dans le Monitorul Oficial no. 18 de 5 Janvier 2011. La loi mise à jour peut être consultée à http://legislatie.just.ro/Public/DetaliiDocument/125150.

${ }^{36}$ La charte peut être consultée à https://unibuc.ro/wp-content/uploads/2018/12/CARTA-UB.pdf, visité le 25 Octobre 2020.
} 
normes de référence au code d'éthique adopté par cette université. Sans analyser les dispositions établies par chaque université roumaine, cela nous permet de souligner que, d'habitude, dans une telle organisation, les normes éthiques fonctionnent dans une architecture de type "norme juridique - norme juridique - norme éthique”.

Comme nous l'avons dit ci-dessus, une telle dynamique ne peut pas exister si les personnes concernées n'ont pas l'obligation juridique de respecter les règles de nature éthique. II en résulte qu'on devrait avoir dans la Charte une ou plusieurs dispositions à cet égard. En effet, art. 23 par. 2 établit que les étudiants ont toujours l'obligation de respecter les normes éthiques, pendant que art. 24 par. 2 du même document mentionne que le personnel enseignant et de recherche a l'obligation de "respecter en toute circonstance les standards de l'éthique universitaire". Donc, à notre avis, les conditions préalable (structurelles) pour avoir un code déontologique performant sont réunies par l'Université de Bucarest. Est-ce que nous sommes d'avis que I'Université de Bucarest a un code déontologique vraiment applicable? Malheureusement, seulement en partie et nous expliquerons notre position dans les paragraphes suivants.

Le Code éthique de l'Université de Bucarest ${ }^{37}$, ci-après dénommé C.E.U.B., régit les situations qui peuvent apparaître dans la vie universitaire tant par des normes avec un haut degré de généralité, que par des normes plus spécifiques. Comme un exemple de norme qui fait partie de la première catégorie on peut citer article 4 e) du C.E.U.B. ; "I'université adopte, par ses structures et par ses membres, une position ferme de prévention de l'abus de pouvoir, d'une part, et de l'abus de confiance, d'autre part." II est facile de voir que cette phrase est plutôt une déclaration qu'une norme de n'importe quelle nature, mais cela ne signifie pas que c'est une erreur d'avoir ce type de normes dans un code déontologique. Elles sont vraiment nécessaires et elles ont un rôle complémentaire parce qu'elles montrent l'intention de l'auteur du texte, donc elles guident le processus d'interprétation. Si le code contient seulement ce type de normes, cela pourrait être un problème dans les situations où le code doit offrir des solutions pour des conduites spécifiques. Heureusement, le C.E.U.B. a aussi des normes éthiques moins générales. Un exemple est l'article 12 qui établit que „l'existence des contributions originales dans une certaine œuvre ou travail ne décharge de responsabilité morale et professionnelle son auteur, si, outre ces contributions, il a inclus aussi dans le travail certain fragments (non attribués d'une manière satisfaisante) dont la présence constitue une violation de la propriété intellectuelle."

En plus, il faut mentionner que le C.E.U.B. contient des normes éthiques pour tous les sujets identifiés au cadre de l'article 130 de la Loi pour l'Education Nationale. Si ces normes sont enfreintes, art. 42 du Code établit qu'on peut appliquer les sanctions prévues par la Charte et par la Loi suscitées (si la violation a été commise par un salarié) ou par le Règlement pour l'activité professionnelle (si la violation a été commise par un étudiant).

Malheureusement, le C.E.U.B., comme beaucoup des autres codes déontologiques cités au cadre de cet article, présente trois problèmes. Premièrement, il utilise plusieurs notions très importantes pour l'économie de l'acte, mais il ne les définit pas. Deuxièmement, le Code n'a plus de quelques règles „originales”, c'est-à-dire qu'il y a peu de règles qui ne se trouve pas déjà dans les actes juridiques applicables. Donc, il y a seulement

${ }^{37}$ On peut consulter le code à https://unibuc.ro/wp-content/uploads/2018/12/Codul-de-Etica\%CC\%86-alUniversit\%CC\%86t\%CC\%A6ii-din-Bucures\%CC\%A6ti.pdf, visité le 23 Décembre 2020. Nous mentionnons que à l'heure de la rédaction du présent article, la dernière édition du Code n'a pas été publiée sur le site de l'Université. Par conséquent, l'édition qu'on peut trouver à cette adresse est seulement un exemple. 
quelques normes pour lesquelles on a besoin d'invoquer le C.E.U.B. et même dans ces situations il est possible de rencontrer des problèmes à cause des notions moins ou pas du tout définies. Troisièmement, il nous manque un mécanisme clairement régit que peut être utilisé par les destinataires des normes éthiques afin de poser des questions dans les situations où un problème de nature éthique pourrait encore être prévenu. Si la norme déontologique n'est pas claire et le destinataire de la norme n'a pas les connaissances nécessaires pour l'interpréter, comment pourrait-on le sanctionner légalement pour l'avoir enfreint au cadre d'un rapport de travail ?

\section{Conclusions}

Comme nous l'avons indiqué dans l'introduction, au lieu de conclusions nous voulons proposer quelques idées que nous pensons être utiles pour l'amélioration de l'architecture normative utilisée actuellement par les universités. Comme nous avons déjà dit plusieurs fois que les normes éthiques doivent être spécifiques et ne doivent pas se limiter à reproduire le contenu des normes juridiques, il nous reste seulement trois idées à développer.

Premièrement, nous suggérons qu'il serait utile de souligner quelles sont les fautes graves (abateri disciplinare grave) tant dans la Charte de I'Université (ou dans un autre acte juridique avec un rôle similaire) que dans le Code déontologique. Il faut mentionner que le droit de travail roumain établit qu'une faute grave pourra mettre fin à un contrat de travail ${ }^{38}$. Comme toutes ces normes peuvent conduire à l'application d'une sanction très sévère par rapport aux dispositions du droit de travail, il est nécessaire de créer un lien non équivoque entre la norme juridique qui contient la sanction et la norme éthique dont le non-respect peut entraîner l'application de ladite sanction. Par ailleurs, il convient que la Commission d'Ethique puisse argumenter que la violation répétée d'une ou de plusieurs normes déontologiques représente une raison pour mettre fin au contrat de travail.

L'idée centrale est la création d'un lien très clair et correct du point de vue juridique entre le Code roumain de travail, la Loi pour l'Education Nationale, les actes juridiques adoptés par les universités (comme les chartes et les règlements) et les codes d'éthique. Comme nous avons déjà dit dans cet article, si on veut avoir un code déontologique applicable aux relations concrètes, on doit établir une connexion entre la sanction de nature juridique, qui est prévue toujours par une norme juridique, et la norme éthique. En absence d'une telle construction, en cas de non-respect de la norme éthique on peut appliquer des sanctions de nature morale, culturelle etc., mais sans le concours du droit.

Il faut préciser qu'il est possible d'avoir une autre situation, plus commune dans les universités roumaines à ce moment. On a un lien entre le Code d'éthique et les normes juridiques, mais ce lien n'est pas du tout non équivoque. A notre avis, un tel lien est comme si ce n'était pas du tout. Au cadre de la procédure disciplinaire l'employeur doit être capable de justifier chaque élément invoqué contre l'employé. Si la base juridique est équivoque, la décision de la commission disciplinaire devient très difficile à expliquer devant le juge.

Deuxièmement, nous croyons qu'il faut avoir une base pour virer les personnes qui ont commis intentionnellement des délits et des crimes (connus en Roumanie comme

\footnotetext{
${ }^{38}$ Conformément à l'article 61 a) du Code roumain de travail. On peut consulter le code de travail à http://legislatie.just.ro/Public/DetaliiDocument/41627, visité le 29 Décembre 2020.
} 
infracțiuni). Une telle règle devrait exister tant dans les documents juridiques que dans les codes d'éthiques. La raison de notre suggestion est que le droit pénal, ainsi que le droit constitutionnel, protègent au niveau national les plus importantes valeurs de la société. Quand une personne viole intentionnellement une norme de droit pénal, en fait elle méprise ces valeurs d'une manière très grave. A notre avis, il n'importe pas si la personne en cause fait partie du personnel enseignant ou du personnel de recherche. En outre, il n'est pas important si la personne a commis le délit/la crime en qualité de collaborateur de l'université ou au cadre de sa vie personnelle. Si la personne a commis l'acte intentionnellement et si on a une décision définitive de condamnation, la Commission d'Ethique doit avoir une base pour recommander le renvoi de la personne concernée.

Il convient aussi d'éviter les formules qui visent à relativiser la gravité de certaines violations de la norme pénale. Par exemple, art. 22 par. (1) a) de la Charte de I'Université établit, inter alia, qu'une personne condamnée est indigne de faire partie du personnel d'enseignement ou de recherche si l'acte commis est "de nature de porter atteinte au prestige académique". Si le droit pénal protège seulement les plus importantes valeurs de la société $^{39}$, est-ce qu'on peut affirmer en toute bonne foi qu'il y a des actes intentionnellement commis qui sont contraires à ses règles, mais qui ne porte pas atteinte au prestige académique ? Est-ce qu'on peut mettre dans la même phrase le prestige académique et le casier judiciaire ? Nous sommes d'avis que non.

Troisièmement, nous croyons qu'il convient d'introduire l'institution d'amicus curiae dans l'activité de la commission d'éthique (ou d'autre organisme similaire). Traditionnellement, les commissions d'éthiques sont composées de membres du personnel d'enseignement ou de recherche. Ces personnes sont très compétentes dans leur domaine d'activité, mais elles seulement rarement ont les connaissances nécessaires pour juger une violation d'une norme éthique dans un cadre bien compliqué du point de vue juridique. On doit s'attendre à des problèmes juridiques et éthiques très complexes, notamment lorsque la violation de la norme éthique a été commise en dehors de la vie académique.

L'utilisation de l'amicus curiae permettrait à la commission d'éthique d'obtenir rapidement et efficacement l'expertise nécessaire pour la résolution de la cause. Un tel mécanisme aiderait aussi la personne accusée, étant donné qu'elle pourrait l'utiliser facilement à son profit. En fait, la commission pourrait demander l'opinion d'une certaine personne soit d'un certain organisme professionnel ou elle pourrait recevoir l'opinion volontaire de n'importe quelle personne avec expertise ou connaissances dans un domaine d'intérêt pour le jugement. Bien sûr, la procédure doit être détaillée afin d'établir quelles sont les limites temporelles, comment est-ce qu'on peut recevoir l'opinion du point de vue technique, comment est-ce qu'on peut poser des questions supplémentaires etc.

\footnotetext{
${ }^{39}$ Constantin Mitrache, Cristian Mitrache, Drept penal român. Partea generală, troisième édition, Universul Juridic, Bucarest, 2019.
} 